\title{
Lactate production and exercise-induced metabolic acidosis: guilty or not guilty?
}

\section{To the Editors:}

In the excellent and very interesting chapter concerning clinical exercise testing published in the European Respiratory Monograph on lung function testing, RocA and RABINOVITCH [1] wrote the following. "Increased lactate production is responsible for the fall in muscle $\mathrm{pH}$, which in turn may play a role in determining exercise intolerance in these patients (COPD patients). Premature lactic acidosis during exercise in COPD patients has been associated with reduced oxidative enzyme concentrations in the lower limb muscles."

We would like to emphasise that the widespread belief that lactic acid is produced in working muscles as a result of oxygen insufficiency, and that it directly causes exerciseinduced metabolic acidosis, has been criticised. In addition, numerous findings on different species are discordant with the prevailing theory [2].

We all accept that when looking at the end result of metabolism during intense exercise, the observed metabolic acidosis coincides with an accumulation of lactate, but does it mean that lactate production is guilty?

Recently, combining a large series of papers, RoBERGS et al. [3] have presented convincing empirical observations that lactate is a consequence rather than a cause of cellular events that cause acidosis.

From the transformation of a glucose molecule to two pyruvate molecules, three reactions release four protons, and one reaction driven by pyruvate kinase consumes two protons. The conversion of two pyruvate molecules to two lactate molecules by lactate dehydrogenase (LDH) consumes two protons. Another source of protons is adenosine triphosphate (ATP) hydrolysis.
During high-intensity exercise, as underlined by ROBERGS et al. [3], there is an increasing dependence on ATP supplied by glycolysis. Under these conditions, there is a greater rate of cytosolic proton release from glycolysis and ATP hydrolysis. The cell buffering capacity can be exceeded and acidosis develops. As a consequence of the favourable bioenergetics for the LDH reaction, lactate production increases. Lactate production does not seem to cause the acidosis and, furthermore, appears to play the role of a "sink" for protons produced during catabolism and ATP hydrolysis, thereby reducing acidosis.

In conclusion, due to "iso-time" apparition of lactate and metabolic acidosis, it remains that for clinical application lactate accumulation is obviously a good, indirect marker of cellular metabolic acidosis.

\section{A. van Meerhaeghe* and B. Velkeniers ${ }^{\#}$}

*Service de Pneumologie, CHU de Charleroi, Hôpital A. Vésale, Montigny-le-Tilleul, and "Dept of Internal Medicine AZ-VUB, Brussels, Belgium.

\section{REFERENCES}

1 Roca J, Rabinovich R. Clinical exercise testing. In: Gosselink R, Stam H, eds. Lung Function Testing. Eur Respir Mon 2005; 31: 146-165.

2 Brooks GA, Dubouchaud H, Brown M, Sicurello JP, Butz CE. Role of mitochondrial lactic dehydrogenase and lactate oxidation in the "intra-cellular lactate shuttle". Proc Natl Acad Sci USA 1999; 96: 1129-1134.

3 Robergs R, Ghiasvand F, Parker D. Biochemistry of exercise-induced metabolic acidosis. Am J Physiol Regul Integr Comp Physiol 2004; 287: R502-R516.

DOI: 10.1183/09031936.05.00059005

\section{Activation of human lung mast cells by monomeric immunoglobulin $\mathrm{E}$}

\section{To the Editors:}

Immunoglobulin (Ig) preparations contain variable proportions of Ig aggregates [1], which behave like true immune complexes and are able to activate inflammatory cells by crosslinking Fc receptors [2]. The validity of the study by CRUSE et al. [3], which appeared in a previous issue of the European Respiratory Journal and showed that monomeric IgE activates 